\title{
Hubert Gollek
}

Über den Orbitraum Liescher Transformationsgruppen

Czechoslovak Mathematical Journal, Vol. 26 (1976), No. 2, 252-258

Persistent URL: http://dml.cz/dmlcz/101396

\section{Terms of use:}

(C) Institute of Mathematics AS CR, 1976

Institute of Mathematics of the Czech Academy of Sciences provides access to digitized documents strictly for personal use. Each copy of any part of this document must contain these Terms of use.

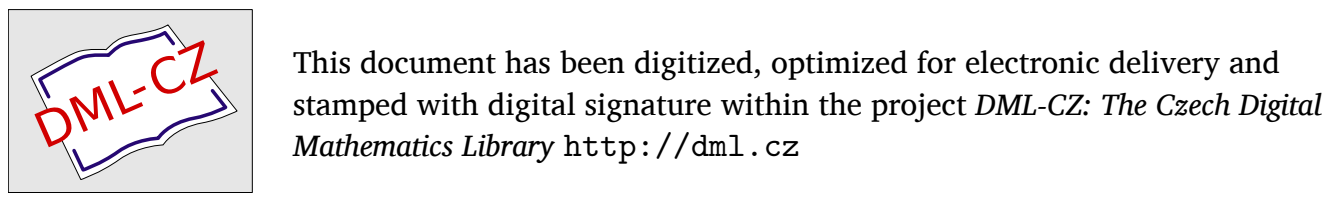




\title{
ÜBER DEN ORBITRAUM LIESCHER TRANSFORMATIONSGRUPPEN
}

\author{
Hubert Gollek, Berlin
}

(Eingegangen am 8. October 1974)

\section{EINLEITUNG}

Ist $G$ eine Liesche Gruppe, die von der Klasse $C^{s}(s \geqq 2)$ auf der zusammenhängenden $C^{s}$-Mannigfaltigkeit $X$ operiert, so wollen wir diese Wirkung mit $(g, x) \in$ $\in G \times X \rightarrow g x \in X$ bezeichnen. Ferner bezeichnen wir für $x \in X$ mit $G x$ das Orbit von $x$, mit $G_{x}$ die Isotropiegruppe von $x$ und mit $\pi_{x}: G \rightarrow G x, \psi_{x}: G \rightarrow G / G_{x}$ bzw. $\varphi_{x}: G / G_{x} \rightarrow G x$ die kanonischen Abbildungen. Dann gilt $\varphi_{x} \circ \psi_{x}=\pi_{x}$ und $\varphi_{x}$ ist eine stetige Bijektion.

Wenn auf dem Orbitraum $X / G$ eine Mannigfaltigkeitsstruktur der Klasse $C^{s}$ derart existiert, daß die kanonische Projektion $\pi: X \rightarrow X / G$ eine Submersion ist, so diese Struktur eindeutig bestimmt und man nennt $X / G$ dann die Orbitmannigfaltigkeit von $(G, X)$.

Für jedes $x \in X$ nennt man die Klasse aller zu $G_{x}$ in $G$ konjugierten Untergruppen den Orbittyp von $x$. In [2] wird bewiesen, daß für jede abgeschlossene Untergruppe $H \subset G$, wenn $G$ kompakt ist, die Menge $X_{(H)}=\{x \in X ; H \in$ Orbittyp von $x\}$ eine (unter Umständen leere) invariante Untermannigfaltigkeit von $X$ ist, für die die Orbitmannigfaltigkeit $X_{(H)} / G$ existiert. Überdies existiert eine Untergruppe $H \subset G$ derart, daß $X_{(H)}$ eine überall dichte, offene Teilmenge ist. Die in $X_{(H)}$ enthaltenen Orbits heißen Hauptorbits und die Klasse der zu $H$ konjugierien Untergruppen heißt der Hauptorbittyp.

Wir zeigen in dieser Arbeit, daß es für jede Liesche Transformationsgruppe $(G, X)$ unter recht schwachen Voraussetzungen in $X$ eine offene, überall dichte, invariante Teilmenge $M(G, X)$ gibt, deren Orbitmannigfaltigkeit $M(G, X) / G$ existiert.

Dazu setzen wir nur voraus, daß alle Orbits lokal abgeschlossen sind.

\section{ORBITS MAXIMALER DIMENSION}

In diesem Paragraphen beweisen wir zwei nicht sehr tief liegende Eigenschaften Liescher Transformationsgruppen. Die bisher eingeführten Bezeichnungen behalten Gültigkeit. 
Satz 1. Es sei $l: X \rightarrow R$ die Funktion $l(x)=\operatorname{dim}(G)-\operatorname{dim}\left(G_{x}\right)$ und $l_{0}=$ $=\max _{x \in X}\{l(x)\}$. Dann ist $M={ }_{\mathrm{Df}} l^{-1}\left(l_{0}\right)$ in $X$ offen. Ist überdies die Wirkung von $G$ auf $X$ analytisch und $X$ zusammenhängend, so ist $M$ in $X$ überall dicht.

Zum Beweis benutzen wir zwei Hilfssätze.

Hilfssatz 1. Sei $X$ eine Mannigfaltigkeit der Klasse $C^{s}(s \geqq 1)$. Seien $E \rightarrow X$ und $F \rightarrow X$ zwei $C^{s}$-Vektorraumbündel, sei $r: E \rightarrow F$ ein $X$-Morphismus der Klasse $C^{s}$ und $r_{x}: E_{x} \rightarrow F_{x}$ für jedes $x \in X$ dessen Einschränkung auf die Fasern. Sei $M_{k}=\left\{x \in X ; \operatorname{dim}\left(\operatorname{Ker}\left(r_{x}\right)\right) \leqq k\right\}$. Dann ist $M_{k}$ offen in $X$.

Beweis. Wir betrachten die kanonische Abbildung

$$
\begin{gathered}
h_{k}: \operatorname{Hom}(E, F) \rightarrow \operatorname{Hom}(\wedge E, \wedge F) \\
h_{k}(A)\left(x_{1} \wedge \ldots \wedge x_{k}\right)=_{\text {Df }} A x_{1} \wedge \ldots \wedge A x_{k} \text { für } \quad x_{i} \in E_{x}, \quad A \in \operatorname{Hom}\left(E_{x}, F_{x}\right) .
\end{gathered}
$$

Diese ist von der Klasse $C^{s}$. Es gilt

$$
M_{k}=\left(h_{n-k} \circ r\right)^{-1}\left(\operatorname{Hom}(\stackrel{n-k}{\Lambda} E, \stackrel{n-k}{\bigwedge} F)-O_{n-k}(X)\right),
$$

wobei $n=\operatorname{dim}(E)$ gesetzt und $r$ als Schnitt über $X$ in $\operatorname{Hom}(E, F)$ betrachtet wird. $O_{n-k}$ bezeichnet den Nullschnitt von $\operatorname{Hom}\left(\wedge^{n-k} E, \stackrel{n-k}{\wedge} F\right)$, q.e.d.

Hilfssatz 2. Die Voraussetzungen seien wie im ersten Hilfssatz. Speziell seien $E, F, r$ und $X$ jetzt analytisch. Wir setzen $k_{0}=\min \left\{k \in Z ; M_{k} \neq \emptyset\right\} . X$ sei zusammenhängend. Für alle $k \geqq k_{0}$ ist dann $M_{k}$ in $X$ überall dicht.

Beweis. Es sei $k \geqq k_{0}$. Wir haben $X-M_{k}=\left\{x \in X ; h_{n-k} \circ r(x)=O_{n-k}(x)\right\}$. Da $X, E, F, r$ von der Klasse $C^{(\omega)}$ sind, sind auch $h_{n-k} \circ r$ und $O_{n-k}$ von der Klasse $C^{\omega}$. Besäße $X-M_{k}$ einen inneren Punkt $x$, so würden $h_{n-k} \circ r$ und $O_{n-k}$ auf der Zusammenhangskomponente von $x$ in $X$ übereinstimmen. Da $X$ zusammenhängend ist, wäre dann $h_{n-k} \circ r=O_{n-k}$ und folglich $M_{k}=\emptyset$. Wegen $M_{k} \supseteq M_{k_{0}}$ steht das im Widerspruch zur Wahl von $k_{0}$, q.e.d.

Beweis von Satz 1. Wir wenden die soeben bewiesenen Hilfssätze auf $E=$ $=X \times \mathfrak{g}(\mathfrak{g}=$ Lie-Algebra von $G)$ und $T(X)$ (Tangentialbündel von $X)$ an. Ist $r$ die Abbildung

$$
r:(x, \mathrm{t}) \in X \times\left.\mathfrak{g} \rightarrow \frac{\mathrm{d}}{\mathrm{d} t} \exp (t \mathrm{t}) x\right|_{t=0} \in T(X),
$$

so its $r$ von der Klasse $x C^{s-1}$ (wobei $\propto-1=\infty, \omega-1=\omega$ zu setzen ist). Wegen $\operatorname{dim}\left(\operatorname{Ker}\left(r_{x}\right)\right)=\operatorname{dim}\left(G_{x}\right)=\operatorname{dim}(G)-l(x)$ für alle $x \in X$ ist $M_{\operatorname{dim}(G)-k}=\{x ;$ $l(x) \geqq k\}$ für alle natürlichen Zahlen $k$, also insbesondere $M_{\operatorname{dim}\left(G_{1}-l_{0}\right.}=M$. Daher 
ist $M$ in $X$ offen. Überdies ist $\operatorname{dim}(G)-l_{0}=\min \left\{k ; M_{k} \neq \emptyset\right\}$, woraus die zweite Behauptung von Satz 1 folgt, q.e.d.

Beis piel 1. Für $s \leqq \infty$ ist die zweite Behauptung von Satz 1 im allgemeinen falsch. Sei $G$ die additive Gruppe der reellen Zahlen, $X=R^{2}$ und $\lambda: R \rightarrow R$ eine $C^{s}$-Funktion mit dem Träger $[-1,1]$. Dann ist durch $(t,(x, y)) \in R \times R^{2} \rightarrow(x, y+\lambda(x) t) \epsilon$ $\in R^{2}$ eine Wirkung der Klasse $C^{s}$ von $R$ auf $R^{2}$ gegeben. Für $x \in(-1,1) \times R$ ist $l(x)=1$ und $l(x)=0$ sonst.

Beis piel 2. Sei $\dot{R}$ die additive Gruppe der reellen Zahlen, versehen mit der diskreten Topologie, $G$ die Liesche Gruppe $R \times \dot{R}$ und $X=R^{2} . G$ operiere auf $X$ durch die gewöhnliche Addition des $R^{2}$. Diese Wirkung ist dann analytisch, transitiv und frei. Dieses Beispiel zeigt, daß im allgemeinen $\operatorname{dim}(G x) \geqq l(x)$ gilt. Gleichheit tritt jedoch immer ein, wenn $G$ separabel ist.

\section{AUSGEZEICHNETE KARTEN}

Wir werden von dieser Stelle an voraussetzen, daß die in $\S 2$ eingeführte Funktion $l(x)$ auf $X$ konstant ist. Dieser konstante Wert sei $k$. Unter dieser Voraussetzung ist

(i) durch $x \in X \rightarrow \Delta(x)={ }_{\text {Df }}\left(\mathrm{d} \pi_{x}\right)_{e}(\mathrm{~g})=r_{x}(\mathrm{~g}) \subset T_{x}(X)$ ein involutives Richtungsfeld der Dimension $k$ gegeben.

(ii) Die Abbildung $\varphi_{x}: G / G_{x} \rightarrow G x$ eine injektive Immersion und $\varphi_{x}(K)$ für jede Zusammenhangskomponente $K$ von $G / G_{x}$ eine maximale Integralmannigfaltigkeit von $\Delta$.

(iii) auf $X$ eine sogenannte Blätterungtopologie dadurch definiert, daß wir eine Teilmenge von $X$ offen nennen, wenn ihr Durchschnitt mit jeder maximalen Integralmannigfaltigkeit von $\Delta$ in dieser offen ist.

(iv) diese Blätterungstopologie die gröbste, für die alle Abbildungen $\varphi_{x}$ offen sind.

Definition 1. Eine Karte $(U, \varphi)$ auf $X$ heißt augezeichnet, wenn folgendes gilt:

1. $\varphi(U)=B^{n}$, wobei wir mit $B_{r}^{m}$ für jede natürliche Zahl $m$ und jede positive reelle Zahl $r$ die Teilmenge $\left\{\left(a^{1}, \ldots, a^{m}\right) ;\left|a^{i}\right|<r, i \in\{1, \ldots, m\}\right\}$ des $R^{m}$ und mit $B^{n}$ die Menge $B_{1}^{n}$ bezeichnen.

2. Die maximalen Integralmannigfaltigkeiten des durch $\Delta$ auf $U$ induzierten Richtungsfeldes entsprechen bei $\varphi$ umkehrbar eindeutig den durch $a^{i}=$ const für $i \in\{1, \ldots, n-k\}$ definierten Untermannigfaltigkeiten von $B^{n}$.

Es sei $R^{n-k}$ mit dem Unterraum der $n$-tupel $\left(a^{1}, \ldots, a^{n-k}, 0, \ldots, 0\right)$ des $R^{n}$ und $R^{k}$ mit dem orthogonalen Komplement (bezüglich des Skalarproduktes $\langle a, b\rangle=\sum_{i=1}^{n} a_{i} b_{i}$ für $\left.a=\left(a^{1}, \ldots, a^{n}\right), b=\left(b^{1}, \ldots, b^{n}\right)\right)$ von $R^{n-k}$ identifiziert. Mit $\pi: R^{n} \rightarrow R^{n-k}$ bezeichnen wir die zugehörige Projektionsabbildung. Dann ist 2) zu der folgenden 
Bedingung äquivalent: Ein Punkt $y \in U$ liegt genau dann in der Zusammenhangskomponente eines anderen Punktes $x$ bezüglich der durch die Blätterungstopologie auf $U$ induzierten Topologie, wenn $\pi \circ \varphi(y)=\pi \circ \varphi(x)$ gilt.

Für jede ausgezeichnete Karte $(U, \varphi)$ ist auf $B^{n-k}$ eine Äquivalenzrelation $R_{U}$ folgendermaßen definiert: Zwei Punkte $a, b \in B^{n-k}$ sind genau dann bezüglich $\boldsymbol{R}_{\boldsymbol{U}}$ äquivalent, wenn $\varphi^{-1}(a)$ und $\varphi^{-1}(b)$ das gleiche Orbit haben. Für $a \in B^{n-k}$ bezeichnen wir mit $R_{U}(a)$ die Äquivalenzklasse von $a$. Es gilt $R_{U}(a)=\pi \circ \varphi(G x \cap U)$, wenn $\pi \circ \varphi(x)=a$ ist.

Offenbar existiert unter unseren Voraussetzungen für jeden Punkt $x \in X$ und jede offene Umgebung $V$ von $x$ eine ausgezeichnete Karte um $x$, deren Definitionsbereich in $V$ enthalten ist.

Hilfssatz 3. Es sei $(U, \varphi)$ eine ausgezeichnete Karte. Für jedes Paar von Punkten $a, b \in B^{n-k}$ mit $R_{U}(a)=R_{U}(b)$ existiert in $B^{n-k}$ ein Paar von Umgebungen $V_{1}, V_{2}$ von $a$ bzw. $b$ und ein Homöomorphismus $\chi_{a, b}: V_{1} \rightarrow V_{2}$ derart, daß $\chi_{a, b}(a)=\boldsymbol{b}$ und $\chi_{a, b}\left(V_{1} \cap R_{U}(c)\right)=V_{2} \cap R_{U}(c)$ für jedes $c \in B^{n-k}$ gilt.

Beweis. Es sei $B_{r}^{n}(a)$ die um den Vektor $a$ verschobene Menge $B_{r}^{n}$ und $g \in G$ ein Element mit $g \circ \varphi^{-1}(a)=\varphi^{-1}(b)$. Es existiert ein $\varepsilon>0$ derart, daß $g \circ \varphi^{-1}\left(B_{\varepsilon}^{n}(a)\right) \subset$ $\subset U$ gilt. Es sei $U_{1}=\varphi^{-1}\left(B_{\varepsilon}^{n}(a)\right)$ und $U_{2}=g\left(U_{1}\right)$. Dann ist $g: U_{1} \rightarrow U_{2}$ eine Isomorphie der beiden auf $U_{1}$ bzw. $U_{2}$ induzierten Blätterungen $\left.\Delta\right|_{U_{1}}$ und $\left.\Delta\right|_{U_{2}}$.

Wir setzen $V_{1}=B_{\varepsilon}^{n-k}(a)=B^{n-k} \cap B_{\varepsilon}^{n}(a)=\pi\left(B_{\varepsilon}^{n}(a)\right), V_{2}=\pi \circ \varphi\left(U_{2}\right)$ und wählen für $\chi_{a, b}$ die Abbildung $c \in V_{1} \rightarrow \pi \circ \varphi \circ g \circ \varphi^{-1}(c) \in V_{2}$. Diese genüg tdann den obigen Forderungen.

Wir zeigen z. Bsp., daß $\chi_{a, b}$ injektiv ist: Sind $c_{1}$ and $c_{2}$ aus $V_{1}$ und ist $\chi_{a, b}\left(c_{1}\right)=$ $=\chi_{a, b}\left(c_{2}\right)$, so liegen $g \circ \varphi^{-1}\left(c_{1}\right)$ und $g \circ \varphi^{-1}\left(c_{2}\right)$ in demselben Blatt von $\left.\Delta\right|_{U_{2}}$. Da aber $g$ eine Isomorphie der beiden Blätterungen $\left.\Delta\right|_{U_{1}}$ und $\left.\Delta\right|_{U_{2}}$ ist, folgt hieraus, daß $\varphi^{-1}\left(c_{1}\right)$ und $\varphi^{-1}\left(c_{2}\right)$ im gleichen Blatt von $\left.\Delta\right|_{U_{1}}$ liegen. Da die Blätter von $\left.\Delta\right|_{U_{1}}$ die Durchschnitte von $U_{1}$ mit den Blättern von $\Delta_{\mid U}$ sind, folgt hieraus $c_{1}=c_{2}$. Die Stetigkeit und die Surjektivität folgen unmittelbar aus der Definition von $\chi_{a, b}$, ebenso die Tatsache, daß $\chi_{a . b}$ offen ist. Um die Gleichung $\chi_{a, b}\left(R_{U}(c) \cap V_{1}\right)=$ $=R_{U}(c) \cap V_{2}$ zu beweisen, genügt der Hinweis, daß für alle $c \in V_{1}$ die Beziehung $\chi_{a, b}(c) \in R_{U}(c)$ gilt, wie man leicht aus $R_{U}(c)=\pi \circ \varphi\left(G \varphi^{-1}(c) \cap U\right)$ schlußfolgert, q.e.d.

Hilfssatz 4. Es sei $(U, \varphi)$ eine ausgezeichnete Karte um $x$. Gx $\cap U$ sei in $U$ abgeschlosen. Ist $G$ separabel, so ist die Menge $A={ }_{\mathrm{Df}} \pi \circ \varphi(G x \cap U)$ diskret.

Beweis. $A$ ist wegen $B^{n-k}-A=\pi \circ \varphi(U-G x)$ abgeschlossen, denn die Abbildung $\pi \circ \varphi$ ist offen. Wir zeigen, daß unter der Voraussetzung, daß $A$ einen Häufungspunkt $a \in A$ besitzt, auch jeder andere Punkt $b \in A$ Häufungspunkt von $\boldsymbol{A}$ ist. In der Tat, es gilt $A=R_{U}(a)=R_{U}(b)$. Wählt man $V_{1}, V_{2}$ und $\chi_{a, b}$ gemäß Hilfssatz 3, so ist $\chi_{a, b}$ ein Homöomorphismus von $V_{1} \cap A$ auf $V_{2} \cap A \chi_{a, b}(a)=b$. Ist 
also $a$ ein Häufungspunkt von $A$, so auch $b$. Da aber somit jeder Punkt aus $A$ auch Häufungspunkt von $A$ und $A$ abgeschlossen ist, ist $A$ perfekt und daher überabzählbar. Ist aber $A$ überabzählbar, so ist $\left\{\left(\pi \circ \varphi \circ \pi_{x}\right)^{-1}(a)\right\}_{a \in A}$ ein überabzählbares System offener, paarweise disjunkter Teilmengen von $G$. Da $G$ separabel ist, darf ein solches System nicht existieren, q.e.d.

Hilfssatz 5. Ist $x \in X, G x$ lokal abgeschlossen und $G$ separabel, so ist die Abbildung $\varphi_{x}: G / G_{x} \rightarrow G x$ ein Homöomorphismus, wobei auf Gx die Relativtopologie $z u$ nehmen ist.

Den Beweis dieses Satzes findet man in [4], Kap. 16, $§ 10$.

\section{REGULÄRE PUNKTE}

Einen Punkt $x \in X$ nennen wir regulär, wenn eine ausgezeichnete Karte $(U, \varphi)$ um $x$ derart existiert, daß für jedes $y \in U$ die Menge $G y^{\prime} \cap U$ in der von $\Delta$ induzierten Blätterungstopologie zusammenhängend ist. Gleichbedeutend damit ist die Bedingung, daß sich die Relation $R_{U}$ auf die Gleichheitsrelation reduziert. Wir bezeichnen mit $M(G, X)$ die Menge aller regulären Punkte von $X$. Aus der Definition folgt unmittelbar, daß $M(G, X)$ in $X$ offen ist. Überdies ist $M(G, X)$ invariant. Für jeden regulären Punkt $x$ und jede reguläre Karte $(U, \varphi)$ um $x$ mit $R_{U}(a)=a$ für alle $a \in B^{n-k}$ ist $\left(g U, \varphi \circ g^{-1}\right)$ eine reguläre Karte um $g x$ mit $R_{g L}=R_{L}$.

Satz 2. Ist $(G, X)$ eine Liesche Transformationsgruppe und $G$ separabel, so existiert die Orbitmannigfaltigkeit $X / G$ genau dann, wenn jeder Punkt $x \in X$ regulär ist.

Den Beweis dieses Satzes findet man in [4], Kap. 16, $\$ 10$.

Satz 3. Es sei $G$ eine separable Liesche Gruppe, die von der Klasse $C^{s}(s \geqq 2)$ auf der zusammenhängenden Mannigfaltigkeit $X$ derart operiert, daß alle Orbits dieser Wirkung lokal abgeschlossen sind un gleiche Dimension haben. Dann ist die Menge $M(G, X)$ aller regulären Punkte von $X$ in $X$ dicht.

Beweis. Da alle Orbits gleiche Dimension haben, existiert um jeden Punkt $x \in X$ eine ausgezeichnete Karte. Wir nehmen an, die Menge $X-M(G, X)$ besäße einen inneren Punkt $x$. Dann existiert um $x$ eine ausgezeichnete Karte $(U, \varphi)$ derart, da kein Punkt $y \in U$ regulär ist. Wir bezeichnen mit $f: B_{1 / 2}^{n-k} \rightarrow Z$ die Abbildung, die jedem $a \in B_{1 / 2}^{n-k}$ die Anzahl der Elemente aus $R_{U}(a) \cap B_{1 / 2}^{n-k}$ zuordnet. Da $R_{U}(a) \cap$ $\cap B^{n-k}$ nach Hilfssatz 4 diskret und die Abschließung von $B_{1 / 2}^{n-k}$ kompakt ist, hat $R_{U}(a) \cap B_{1 / 2}^{n-k}$ nur endlich viele Elemente.

Der Umstand, daß alle Punkte $y \in U$ nicht regulär sind, hat zur Folge, daß in jeder Umgebung von $\pi \circ \varphi(y)$ in $B_{1 / 2}^{n-k}$ zwei verschiedene. bezüglich $R_{U}$ äquivalente 
Punkte liegen. Dann liegen in jeder offenen Teilmenge von $B_{1 / 2}^{n-k}$ zwei verschiedene äquivalente Punkte.

Die Funktion $f: B_{1 / 2}^{n-k} \rightarrow Z$ besitzt folgende Eigenschaften:

(i) Jeder Punkt $a \in B_{1 / 2}^{n-k}$ hat in $B_{1 / 2}^{n-k}$ eine offene Umgebung $U_{a}$ derart, daß $f(b) \geqq$ $\geqq f(a)$ für alle $b \in U_{a}$ gilt.

(ii) Für jede offene Teilmenge $A \subset B_{1 / 2}^{n-k}$ ist die Einschränkung $\left.f\right|_{A}$ nicht beschränkt.

Zum Beweis von (i) betrachten wir einen beliebigen Punkt $a \in B_{1 / 2}^{n-k}$. Es sei $f(a)=$ $=m, R_{U}(a) \cap B_{1 / 2}^{n-k}=\left\{a_{1}, \ldots, a_{m}\right\}$ und $a=a_{1}$. Wir wählen gemäß Hilfssatz 3 für jedes $j \in\{2, \ldots, m\}$ ein Paar von Umgebungen $V_{1, j}$ und $V_{j}$ von $a_{1}$ bzw. $a_{j}$ und einen Homöomorphismus $\chi_{a_{1}, a_{j}}=\chi_{j}: V_{1, j} \rightarrow V_{j}$ mit $\chi_{j}\left(a_{1}\right)=a_{j}$ und $R_{U}(b) \cap V_{j}=$ $=\chi_{j}\left(R_{U}(b) \cap V_{1, j}\right)$ für jedes $j \in\{2, \ldots, m\}$ und für alle $b \in B^{n-k}$. Überdies seien alle Mengen des Systems $\left\{V_{1, j}, V_{j}\right\}$ paarweise disjunkt. Wir setzen $U_{a}=\bigcap_{j=2}^{m} V_{1, j}$. Ist $b \in U_{a}$, so gilt $\chi_{j}(b) \in R_{U}(b) \cap V_{j}$ für $j \in\{2, \ldots, m\}$. Wegen $U_{a} \cap V_{j}=\emptyset$ und $V_{i} \cap$ $\cap V_{j}=\emptyset$ für $i \neq j$ sind alle $\chi_{j}(b)$ verschieden. Folglich ist $f(b) \geqq m=f(a)$. Zum Beweis von (ii) behalten wir die soeben eingeführten Bezeichnungen bei. Es genügt zu zeigen, daß in jeder Umgebung eines jeden Punktes $a \in B_{1 / 2}^{n-k}$ ein Punkt $b$ mit $f(b) \geqq 2 f(a)$ existiert. Ist also $V$ eine Umgebung von $a$, so existieren in $V \cap U_{a}$, wie oben erwähnt, zwei verschiedene Punkte $b, c$ mit $b \in R_{U}(c)$. Dann sind die $2 m$ Punkte $b, \chi_{2}(b), \ldots, \chi_{m}(b), c, \chi_{2}(c), \ldots, \chi_{m}(c)$ paarweise verschieden und zueinander äquivalent. Daher gilt $f(b) \geqq 2 m=2 f(a)$. Funktionen, die die Eigenschaften (i) und (ii) gleichzeitig besitzen, existieren jedoch nicht. Wegen (i) ist nämlich für jedes $k \in Z$ die Menge $M_{k}=\left\{a \in B_{1 / 2}^{n-k} ; f(a) \geqq k\right\}$ offen und wegen (ii) ist $M_{k}$ in $B_{1 / 2}^{n-k}$ dicht. Da aber der Durchschnitt eines abzählbaren Systems überall dichter, offener Teilmengen von $B_{1 / 2}^{n-k}$ in $B_{1 / 2}^{n-k}$ dicht ist und da $\bigcap_{k=1}^{\infty} M_{k}=\emptyset$ gelten muß, wie man leicht aus der Definition der $M_{k}$ ersieht, haben wir auf diese Weise einen Widerspruch zu der Annahme erhalten, $X-M(G, X)$ besäße einen inneren Punkt, q.e.d.

Beispiel 3. Wir lassen die Gruppe $Z$ der ganzen Zahlen auf $R^{2}$ durch $(n,(x, y)) \in$ $\in Z \times R^{2} \rightarrow\left(n+x,(-1)^{n} y\right) \in R^{2}$ operieren und identifizieren den Faktorraum $R^{2} / Z$ mit dem Möbiusband. Wir lassen ferner die additive Gruppe $R$ der reellen Zahlen $R$ durch $(t,(x, y) \bmod Z) \in R \times R^{2} / Z \rightarrow(t+x, y) \bmod Z \in R^{2} / Z$ auf $R^{2} / Z$ operieren. Dann ist $(x, y) \bmod Z$ genau dann aus $M\left(R, R^{2} / Z\right)$ wenn $y \neq 0$ gilt.

Beispiel 4. Sei $V$ ein euklidischer Vektorraum und $U \subset V$ ein Unterraum. Die Gruppe $Z_{2}=\{1,-1\}$ operiere auf $V$ durch Spiegelung an $U$. Dann ist $M\left(Z_{2}, V\right)=$ $=V-U$.

Aus den bewiesenen bzw. zitierten Sätzen folgt nun leicht der folgende

Satz 4. G sei eine separable Liesche Gruppe, die analytisch auf der zusammenhängenden, analytischen Mannigfaltigkeit $X$ derart operiert, daß alle Häufungs- 
punkte jedes Orbits Gx maximaler Dimension nicht zu einem von Gx verschiedenen Orbit maximaler Dimension gehören. Dann existiert in $X$ eine offene, überall dichte, invariante, aus Orbits maximaler Dimension bestehende Teilmenge $M(G, X)$, deren Orbitmannigfaltigkeit $M(G, X) / G$ existiert.

Wir weisen darauf hin, daß $M(G, X)$ im allgemeinen kein Faserbündel über $M(G, X) / G$ ist.

\section{Literatur}

[1] J. L. Koszul, Lectures on Groups of Transformations, Tata Institute of Fundamental Research, Bombay 1965.

[2] K. Jänich, Differenzierbare G-Mannigfaltigkeiten, Lecture notes in Mathematics, 59, Springer Verlag 1968.

[3] J. Dieudonné, Éléments d'analyse, Vol III, Paris 1972.

[4] R. Richardson, On the Variation of Isotropy Subalgebras, Proc. of the Conf. on Transf. Groups New Orleans 1967, Springer Verlag 1968, 429-440.

Anschrift des Verfassers: 108 Berlin, Unter den Linden 6, DDR (Sektion Mathematik der Humboldt-Universität). 